\title{
Macaulayite, a new mineral from North-East Scotland
}

\author{
M. J. Wilson, J. D. Russell, J. M. Tait, D. R. Clark, and A. R. Fraser \\ The Macaulay Institute for Soil Research, Craigiebuckler, Aberdeen, Scotland
}

ABSTR ACT. Macaulayite was found by the late I. Stephen (Soil Science Department, University of Aberdeen) in an outcrop of reddened, deeply weathered granite, near Inverurie, Aberdeenshire. It is blood red in colour, very fine-grained, and has refractive indices greater than 1.734 Its calculated density is $4.41 \mathrm{~g} / \mathrm{cm}^{3}$. The mean of fourteen electron microprobe analyses in the anhydrous form is $\mathrm{Fe}_{2} \mathrm{O}_{3} 84.67, \mathrm{Al}_{2} \mathrm{O}_{3} 4.01, \mathrm{SiO}_{2} 11.32 \%$. With thermogravimetric data this leads to a formula of $\left(\mathrm{Fe}_{44.75}^{3+}\right.$ $\left.\mathrm{Al}_{3.38}\right) \mathrm{Si}_{7.95} \mathrm{O}_{86}(\mathrm{OH})_{4}$; the ideal formula is $\mathrm{Fe}_{24}^{3+} \mathrm{Si}_{4} \mathrm{O}_{43}$ $(\mathrm{OH})_{2}$. The cell indexes as $C$-centred monoclinic with $a$ s.038, $b 8.726, c 36.342 \AA, \beta 92^{\circ}$. The strongest $\mathrm{X}$-ray lines are 36.6 (vs), 18.16 (vs), $3.700(25), 2.720$ (35), 2.533 (100), 2.214 (20), and 1.420 (35). Macaulayite has a layer structure, thought to consist of a double hematite unit terminated on both sides by silicate sheets and with water between these sheets. The infra-red spectrum includes absorption bands at $3597,1052,1033$, and $858 \mathrm{~cm}^{-1}$, arising from the hydroxysilicate component of the mineral and at $647,520,438,400,304$, and $227 \mathrm{~cm}^{-1}$ corresponding to the platy hematite unit. The mineral is named for the Macaulay Institute for Soil Research and the name was approved by the Commission on New Minerals and Mineral Names of the International Mineralogical Association prior to publication.

THE occurrence and basic characteristics of this mineral have already been described under the provisional description of a 'swelling hematite/ layer silicate mineral' (Wilson et al., 1981). The account that follows gives the essential data for identification including some that have been obtained since the previous publication

Macaulayite is found in the bright red patches of an outcrop of deeply weathered granite located at the eastern end of the Bennachie car park some $7 \mathrm{~km} \mathrm{WNW}$ of Inverurie, Aberdeenshire (National Grid Reference NJ 682246). Further details are given by Wilson et al. (1981). The mineral has a blood-red colour and an earthy nature. It occurs as fine-grained pigmentary material, widely disseminated throughout the weathered rock and is intimately associated with kaolinite and illite. Macaulayite was concentrated in the $<2 \mu \mathrm{m}$ fraction of the decomposed rock by the usual sedimentation procedures and was further enriched by removal of kaolinite by the HF method described by Norrish (1968). As this treatment removes some silica from the macaulayite structure, however, an electromagnetic separation procedure was devised which was based essentially on the method described by Schulze and Dixon (1979). This method yielded highly enriched material in an unaltered form. All the data that follow were obtained on material separated this way.

$X$-ray and electron diffraction data. An X-ray single crystal study could not be made because of the fine-grained nature of the material. The powder pattern yielded by macaulayite shows a very strong reflection at $36 \AA$ with two lower orders at 18 and $12 \AA$ approximately. These high spacings are best observed by diffractometry using oriented mounts and evidently derive from the basic layer structure. A fundamental $36 \AA$ structure can also be observed using high resolution lattice imaging of resinembedded material (Wilson et al. 1981). The remaining part of the X-ray powder pattern is somewhat similar to that of hematite although several important points of detail should be noted. Thus, the pattern more closely resembles that of so-called 'disordered' hematite, which is the form produced immediately after the dehydration of goethite at $200^{\circ} \mathrm{C}$ (Brown, 1980). All spacings are slightly larger than those of highly crystalline hematite and the pattern also shows a well-marked differential line broadening effect. In addition, the reflections at $4.38,4.20$, and $3.61 \AA$ (fig. 1) have no counterpart in the hematite pattern. Electron diffraction patterns of macaulayite particles can be readily interpreted in terms of an oriented association between hematitelike and layer silicate phases. Thus, the layer silicate component yields a typical hexagonal diffraction pattern with weak 11,02 reflections close to the central beam, but with the 13,20 reflections coinciding with the 110 reflections of hematite. This indicates that the $b$ axis of the silicate lies parallel to the $a_{\text {hex }}$ axis of hematite, a relationship that could account for the sharp reflections noted in the X-ray 


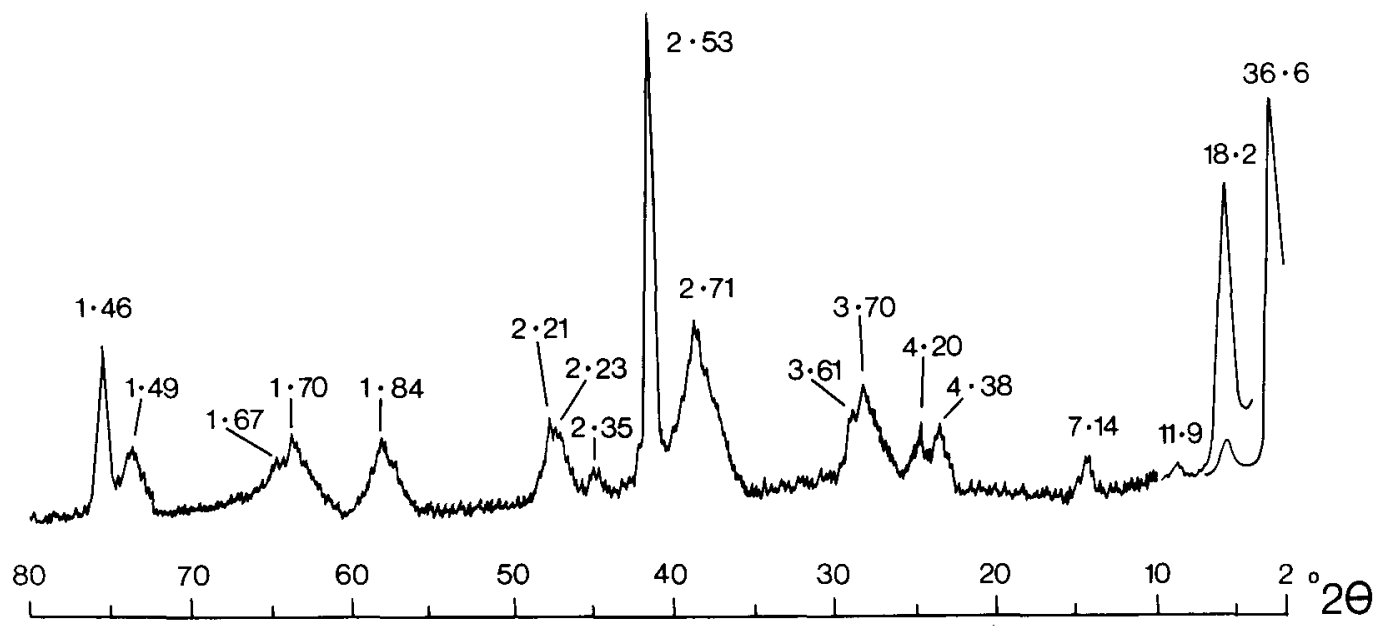

Fig. 1. Diffractometer pattern of macaulayite obtained with $\mathrm{Co}-K \alpha$ radiation, $40 \mathrm{kV} 35 \mathrm{~mA}$, using an oriented aggregate from 2 to $10^{\circ} 2 \theta$ and a randomly ordered powder from 10 to $80^{\circ} 2 \theta$. Divergence, receiving, and antiscatter slits as follows: $2-5^{\circ} 2 \theta, \frac{1}{4}^{\circ}, 0.1 \mathrm{~mm}, \frac{1}{4}^{\circ} ; 5-10^{\circ} 2 \theta, \frac{1}{2}^{\circ}, 0.1 \mathrm{~mm}, \frac{1}{2}^{\circ} ; 10-80^{\circ} 2 \theta, 1^{\circ}, 0.2 \mathrm{~mm}, 1^{\circ}$.

powder pattern. These reflections appear to originate from planes common to both hematite and silicate sheets so that in these directions the structure is well ordered. In other directions, however, no such ordering exists, leading to line broadening. From the $\mathrm{X}$-ray and electron diffraction data, it is concluded that macaulayite has a $C$-centred monoclinic cell. Unit cell parameters and an indexed powder pattern are shown in Table I. An interesting feature of macaulayite is that it is able to accommodate molecules of water, glycerol, or ethylene glycol between its layers leading to an increase of approximately 4 to $5 \AA$ in layer spacing in the same way as is found with smectites. Heating at 300 or $500^{\circ} \mathrm{C}$ leads to a contraction of about $2 \AA$. Fuller details are given in Wilson et al. (1981).

Chemical and thermogravimetric data. The mean of fourteen microprobe analyses of individual macaulayite particles gave a mean $(\mathrm{Fe}+\mathrm{Al}): \mathrm{Si}$ ratio of $6: 1$ and a mean $\mathrm{Fe}: \mathrm{Al}$ ratio of 13.6. These ratios lead to an anhydrous analysis of $\mathrm{Fe}_{2} \mathrm{O}_{3}$ $84.67, \mathrm{Al}_{2} \mathrm{O}_{3} 4.01, \mathrm{SiO}_{2} 11.32 \%$ after recasting to $100 \%$. A thermogravimetric curve of macaulayite showed a total water loss of $7.4 \%$, which was interpreted in terms of adsorbed and interlayer $\mathrm{H}_{2} \mathrm{O}(2.5 \%)$, edge $\mathrm{OH}$ groups and interstitial $\mathrm{H}_{2} \mathrm{O}$ $(3.7 \%)$, and structural $\mathrm{OH}$ groups $(1.2 \%)$. Taking into account structural $\mathrm{OH}$ and the observed $(\mathrm{Fe}+\mathrm{Al}): \mathrm{Si}$ ratio, the formula for macaulayite corresponds to $\left(\mathrm{Fe}_{44.75}^{3+} \mathrm{Al}_{3.38}\right) \mathrm{Si}_{7.95} \mathrm{O}_{86}(\mathrm{OH})_{4}$. The $\mathrm{OH}$ content is derived by analogy with pyrophyllite and the ideal formula for the pure iron end member of macaulayite would be $\mathrm{Fe}_{24}^{3+} \mathrm{Si}_{4}$ $\mathrm{O}_{43}(\mathrm{OH})_{2}$.
Infra-red spectroscopy. The infra-red spectrum of the $<2 \mu \mathrm{m}$ clay and the electromagnetically concentrated fraction from it (fig. $2 a, b$ ) clearly show the dramatic enhancement of absorption bands of

T A B LE I. X-ray powder data for macaulayite, $\mathrm{Co}-K \propto$ radiation

\begin{tabular}{|c|c|c|c|}
\hline$I_{\text {est }}$ & $d_{\text {meas }}$ & $d_{\text {calc }}$ & $h k l$ \\
\hline vs* & 36.6 & 36.32 & 001 \\
\hline vs* & 18.16 & 18.16 & 002 \\
\hline $\mathrm{w}^{*}$ & 11.87 & 12.11 & 003 \\
\hline 15 & 4.377 & $\begin{array}{l}4.363 \\
4.361\end{array}$ & $\left.\begin{array}{c}020 \\
110\end{array}\right\}$ \\
\hline 15 & 4.202 & 4.212 & 112 \\
\hline 25 & 3.700 & 3.690 & 115 \\
\hline 15 & 3.612 & 3.632 & 0.0 .10 \\
\hline 35 & 2.720 & 2.750 & 1.1 .10 \\
\hline 100 v. sharp & 2.533 & $\begin{array}{l}2.519 \\
2.517\end{array}$ & $\left.\begin{array}{c}130 \\
200\end{array}\right\}$ \\
\hline 15 & 2.231 & $\begin{array}{l}2.235 \\
2.230\end{array}$ & $\begin{array}{l}207 \\
0.2 .14\end{array}$ \\
\hline 20 & 2.214 & 2.201 & 1.1 .14 \\
\hline 15 & 1.844 & 1.845 & 2.2 .10 \\
\hline 10 & 1.699 & 1.694 & 2.2 .13 \\
\hline 10 & 1.671 & 1.670 & 0.4 .14 \\
\hline 5 & 1.507 & 1.507 & 319 \\
\hline 10 & 1.493 & 1.495 & 1.5 .10 \\
\hline 35 v. sharp & 1.462 & $\begin{array}{l}1.454 \\
1.453\end{array}$ & $\left.\begin{array}{l}060 \\
330\end{array}\right\}$ \\
\hline
\end{tabular}

Monoclinic, $C$-centred cell $a 5.038, b 8.726, c 36.342 \AA$, $\beta 92^{\circ}, Z=2$.

* Oriented mounts. 


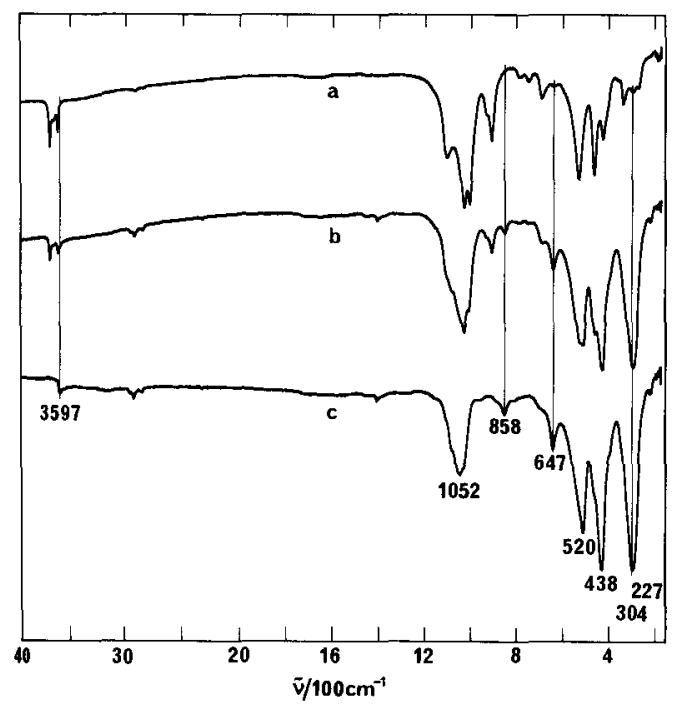

Fig. 2. Infra-red spectra of macaulayite in $(a)$ untreated $<2 \mu \mathrm{m}$ clay with kaolinite impurity, (b) electromagnetically concentrated fraction of $<2 \mu \mathrm{m}$ clay, (c) computer-generated difference spectrum between magnetic concentrate and $<2 \mu$ m clay.

macaulayite at $858,647,520,438$, and $304 \mathrm{~cm}^{-1}$, relative to those of kaolinite which is the major phase in the clay. The spectrum of macaulayite (fig. $2 c$ ) is a computer-generated difference spectrum between the magnetic concentrate and the clay, and corresponds to undistorted spectral data. The spectrum reported previously (Wilson et al., 1981) was an attempt to cancel kaolinite absorption by running magnetic concentrate against an appropriate amount of clay in the spectrometer. Although it was of reasonable quality, the previous spectrum contained residual bands near the kaolinite positions and also an instrumental artefact at 1087 $\mathrm{cm}^{-1}$. The spectrum in fig. $2 c$ is free from these spurious features, and clearly shows the hydroxysilicate-hematite nature of the mineral. The resemblance of the $3597,1052,1033$, and $858 \mathrm{~cm}^{-1}$ bands of the hydroxysilicate component to ferripyrophyllite and of the hematite component to a platy form of this mineral has been dealt with in detail by Wilson et al.(1981). It has been shown subsequently that the perpendicular nature of the hematite bands at 647 and $400 \mathrm{~cm}^{-1}$, i.e. with vibrations parallel to the $c$ axis, are entirely consistent with the platy morphology reported (Rendon and Serna, 1981; Serna et al., 1982) and substantiate the claim for a layer structure for macaulayite.

Optical and other properties. Optically, macaulayite consists of small, sub-angular, platelike particles, less than $2 \mu \mathrm{m}$ in diameter and with a pale-red or yellowish colour. Its refractive index is greater than 1.734. Pleochroism is uncertain because of the fine grain size. A basal cleavage is strongly suggested by the marked orientation effect observed by X-ray diffraction, infra-red spectroscopy, and electron microscope observations. The calculated density of macaulayite is $4.41 \mathrm{~g} / \mathrm{cm}^{3}$.

Structure. X-ray and electron diffraction data strongly suggest that macaulayite has a layer structure. The ideal formula is consistent with an arrangement consisting of twelve $\mathrm{Fe}$ octahedral sheets bounded by two Si tetrahedral sheets, corresponding essentially to a double hematite unit terminated on both sides by silicate sheets. Adjacent basal oxygen surfaces of these sheets are only weakly bonded, allowing structural expansion and the introduction of a layer of water molecules in the interlamellar space. The cation exchange capacity of macaulayite is very low $(<6 \mathrm{~m}$. eq/100 g) suggesting that the charge characteristics of the mineral and the nature of the interlayer space are more akin to halloysite than to smectite or vermiculite.

Possible relationship with melanosiderite. On the basis of its chemical composition, macaulayite appears to be close to the mineral melanosiderite described by Cooke (1875). However, a recent examination of melanosiderite shows that this material consists largely of siliceous ferrihydrite (Wilson and Russell, 1983) and is quite unrelated to macaulayite.

Name. Macaulayite is named after the parent institute of the authors, the Macaulay Institute for Soil Research. Type material will be deposited at the British Museum (Natural History), and in the Royal Scottish Museum.

Acknowledgements. We thank Professor A. Kato, Natural Science Museum, Tokyo, for his help and encouragement.

\section{REFERENCES}

Brown, G. (1980) In Crystal Structures of Clay Minerals and their $X$-ray Identification $(\mathrm{G}$. W. Brindley and G. Brown, eds.). Mineralogical Society, London, 361-410. Cooke, J. P. (1875) Proc. Am. Acad. Arts. Sci. 10, 451-2. Norrish, K. (1968) Trans. 9th Int. Congr. Soil Sci. 111, 713-23.

Rendon, J. L., and Serna, C. J. (1981) Clay Minerals, 16, 375-81.

Schulze, D. G., and Dixon, J. B. (1979) Soil Sci. Soc. Am. J. 43, 793-9.

Serna, C. J., Rendon, J. L., and Iglesias, J. E. (1982) Spectrochim. Acta, 38A, 797-802.

Wilson, M. J., and Russell, J. D. (1983) Mineral. Mag. 47, $85-7$.

- Tait, J. M., Clark, D. R., Fraser, A. R., and Stephen, I. (1981) Clay Minerals, 16, 201-77.

[Manuscript received 14 March 1983] 